\title{
Fatigue life prediction of dentin-adhesive interface using micromechanical stress analysis
}

\author{
Viraj Singh ${ }^{2}$, Anil Misra ${ }^{1,3}$, Orestes Marangos ${ }^{2,3}$, Jonggu Park ${ }^{3}$, Qiang $\mathrm{Ye}^{3}$, Sarah L \\ Kieweg $^{2}$, and Paulette Spencer ${ }^{2,3}$ \\ ${ }^{1}$ Civil, Environmental and Architectural Engineering Department, University of Kansas \\ ${ }^{2}$ Mechanical Engineering Department, University of Kansas \\ ${ }^{3}$ Bioengineering Research Center (BERC), University of Kansas
}

\section{Abstract}

Objectives-The objective of this work was to develop a methodology for the prediction of fatigue life of the dentin-adhesive (d-a) interface.

\begin{abstract}
Methods-At the micro-scale, the d-a interface is composed of dissimilar material components. Under global loading, these components experience different local stress amplitudes. The overall fatigue life of the $\mathrm{d}$-a interface is, therefore, determined by the material component that has the shortest fatigue life under local stresses. Multiple $3 \mathrm{~d}$ finite element (FE) models were developed to determine the stress distribution within the $\mathrm{d}$-a interface by considering variations in micro-scale geometry, material composition and boundary conditions. The results from these models were analyzed to obtain the local stress concentrations within each d-a interface component. By combining the local stress concentrations and experimentally determined stress versus number of cycle to failure (S-N) curves for the different material components, the overall fatigue life of the da interface was predicted.
\end{abstract}

Results-The fatigue life was found to be a function of the applied loading amplitude, boundary conditions, microstructure and the mechanical properties of the material components of the d-a interface. In addition, it was found that the overall fatigue life of the d-a interface is not determined by the weakest material component. In many cases, the overall fatigue life was determined by the adhesive although exposed collagen was the weakest material component. Comparison of the predicted results with experimental data from the literature showed both qualitative and quantitative agreement.

Significance-The methodology developed for fatigue life prediction can provide insight into the mechanisms that control degradation of the bond formed at the d-a interface.

\section{Keywords}

dentin; adhesive; interface; bond; hybrid layer; fatigue; finite element

\footnotetext{
(C) 2004 Academy of Dental Materials. Published by Elsevier Ltd. All rights reserved.

Corresponding Author: Dr. Anil Misra, Professor, Civil, Environmental and Architectural Engineering Department, The University of Kansas, Learned Hall, 1530 W. 15th Street, Lawrence, KS 66045-7609, Ph: (785) 864-1750, Fax: (785) 864-5631, amisra@ku.edu.

Publisher's Disclaimer: This is a PDF file of an unedited manuscript that has been accepted for publication. As a service to our customers we are providing this early version of the manuscript. The manuscript will undergo copyediting, typesetting, and review of the resulting proof before it is published in its final citable form. Please note that during the production process errors may be discovered which could affect the content, and all legal disclaimers that apply to the journal pertain.
} 


\section{INTRODUCTION}

In composite restorations that utilize a two-step adhesive system, dentin is etched with acidic agents to dissolve the mineral component. Dentin adhesive is applied to the etched surface to form a hybrid layer (HL); the hybrid layer is the demineralized dentin matrix infiltrated with adhesive resin. The characteristics of the hybrid layer depend upon the permeability and thickness of the demineralized dentin as well as the diffusibility of the applied adhesive resin. Typically, the hybrid layer has graded properties with depth [1-3]. The thickness of the hybrid layer and amount of adhesive infiltration depends upon the type of adhesive, characteristics of the dentin substrate and operator technique. In some cases, the adhesive will not infiltrate to the depth of the demineralized dentin and under these circumstances, an exposed collagen layer may exist below the hybrid layer [4].

Clearly, the resultant dentin-adhesive (d-a) interface is a complex construct of dissimilar material components. The properties of these material components have a significant effect upon the mechanical performance and durability of the d-a interface. Based upon microscale structure-property measurements, we have previously developed an idealized microstructural representation of the d-a interface $[1,5]$. This idealized model of the d-a interface can be utilized to perform micromechanical finite element $(\mu \mathrm{FE})$ analyses.

The $\mu \mathrm{FE}$ analyses have shown that the each material phase at the d-a interface experiences different stress concentration under functional load $[1,5]$. Since each component of the d-a interface reaches its failure strength at a different stress-level, the overall failure behavior is not determined by the weakest component. Indeed, the component whose stress concentration is closest to its failure strength determines the failure. Therefore, under function, the overall time dependent behavior of the interface is a complex function of the time-dependent behavior of the individual material phases.

In this paper, we have developed 3-dimensional models of the d-a interface and performed the $\mu \mathrm{FE}$ analyses. The resultant stress distributions were analyzed to show the effect of stress concentration on the overall fatigue failure behavior of the $\mathrm{d}$-a interface. The stresses in the different phases of the d-a interface predicted by the $\mu \mathrm{FE}$ were used against S-N (stress-cycle) curves corresponding to each component of the d-a interface [6]. The overall failure strength and S-N curve for the d-a interface were computed. The strength, the endurance limit, and the S-N curve of the d-a interface were found to be a function of the applied loading amplitude, the boundary conditions, the microstructure of the interface and the mechanical properties of the different material phases. The results from our methodology were compared with experimental data from the literature for model verification.

\section{MATERIALS AND METHODS}

\section{Computational Unit Cell Idealization for Micro-scale 3d FE Model}

Based upon the results from micro-Raman spectroscopic, scanning acoustic microscopic and optical microscopic investigations $[2,7,8]$, the $\mathrm{d}$-a interface may be represented as shown in Figure 1. In this idealization the dentin-adhesive interface is considered to be composed of restorative dental composite, adhesive, the hybrid layer, exposed collagen, the partially demineralized dentin, and dentin. As a result, a 3d parallelepiped unit cell shown in Figure 1 with square cross-section is used to represent the d-a interface.

To perform the micromechanical stress analysis a $3 \mathrm{~d}$ linear elastic FE model of the representative unit cell was developed as shown in Figure 2. To reduce the computational effort, quarter symmetry was used in this study. The geometrical properties of the interface components were taken from the literature [1]. The computational unit cell was $37.0 \mu \mathrm{m}$ and 
$8.0 \mu \mathrm{m}$ in longitudinal and transverse sections, respectively. The unit cell is further divided in different volumes to represent individual material components in the d-a interface as shown in Figures 1 and 2. The different material components are: adhesive-collagen composite (hybrid layer), adhesive, restorative dental composite, partially demineralized dentin, peritubular dentin and intertubular dentin. The adhesive appears as a layer underlying the restorative dental composite as well as the adhesive tags formed as the adhesive flows into the dentinal tubules (tubules were opened as a result of acid-etching). Also, the hybrid layer (adhesive-collagen composite) is divided into several layers in order to represent the variation of properties within this layer due to imperfect infiltration of adhesive into the demineralized dentin. The thicknesses (in $\mu \mathrm{m}$ ) of the restorative dental composite, the adhesive layer, and the partially demineralized dentin are 5, 5, and 2.0, respectively. The width of the peritubular dentin varies and the height of the intertubular dentin is $18 \mu \mathrm{m}$. The thickness of the hybrid layer in this study varied from 10 to $5 \mu \mathrm{m}$.

Boundary Conditions for the FE Model-On the two symmetric sides as shown in Figure 2, symmetric boundary conditions were imposed and on the other two sides periodic boundary conditions were imposed. On the plane perpendicular to the Y-axis, the out-ofplane displacement (displacement in Y direction) is zero, i.e. that unit cell is only allowed to deform in the $\mathrm{XZ}$ plane. Similarly on the plane perpendicular to the $\mathrm{X}$-axis, displacement in the $\mathrm{X}$ direction is zero and the unit cell is allowed to deform in the $\mathrm{YZ}$ plane. This means that the unit cell will retain its original cross-section under applied loading in the $\mathrm{Z}$ direction. The bottom surface of the unit cell is constrained in all three directions, i.e. zero displacement in $\mathrm{x}, \mathrm{y}$ and $\mathrm{z}$ direction. The top surface of the idealized unit cell was subject to one of the following types of loading boundary conditions (1) stress controlled consisting of a uniform tensile traction of $20 \mathrm{MPa}$ and (2) strain controlled consisting of displacement compatible with a uniform tensile unit strain.

These simplified boundary conditions are considered because the complex loading conditions at the $\mathrm{d}-\mathrm{a}$ interface precludes modeling of actual boundary conditions. The complex loading condition at the d-a interface is clear from the stress distributions obtained for an example 3-d FE analysis of a restored tooth as shown in Figure 3. For this global analysis, a FEM model of mandibular right 2nd permanent molar was created. Quarter geometry was considered in order to reduce the computational time. The $3 \mathrm{~d}$ global model considered the material component properties as follows: composite $(\mathrm{E}=30 \mathrm{GPa}, \nu=0.30)$, enamel $(\mathrm{E}=70 \mathrm{GPa}, \nu=0.20)$, dentin $(\mathrm{E}=28 \mathrm{GPa}, \nu=0.25)$, and $\mathrm{d} / \mathrm{a}$ interface $(\mathrm{E}=5 \mathrm{GPa}, \nu=0.4)$. The model consists of 716550 solid parabolic tetrahedral elements, of which 141310 elements are within the $100 \mu \mathrm{m}$ thick d-a interfacial region. Discretization refinement was performed to obtain converged results. The bottom boundary nodes of the 3D tooth were constrained in the vertical direction. The nodes on the surface in z-x plane and surfaces in y$\mathrm{z}$ planes were constrained in $\mathrm{x}$ and $\mathrm{y}$ axis respectively. To simulate the physiological patterns of contact during chewing non-homogeneous morsels that contain a combination of hard gum and bone [9], a compressive traction force of $1 \mathrm{~N} / \mathrm{mm}^{2}$ is applied at $45^{\circ}$ to the occlusal surface of the crown. The d-a interface stress distributions from the global model clearly show (Figure 3c) that the actual loading conditions of the computational unit cell will be (i) complex, (ii) depend upon the occlusal loading conditions, and (iii) vary from location to location along the d-a interface. Qualitatively similar d-a interface stress distributions will be obtained for variations of the global model although the quantitative details may be different. Concurrent local-global modeling is too computationally intensive to provide practical, accurate results that capture all the physiologic loading conditions (such as soft versus hard morsels). On the other hand, it is well-known that traction and displacement boundary conditions provide an overall upper and lower-bound behavior of the computational unit-cell [10]. Hence to calculate the bounds of the mechanical behavior of the unit cell under physiologic loading conditions, e.g. chewing, talking, grinding, etc. we 
have studied the behavior of the unit cell under two types of loading boundary conditions (listed above).

Material Properties and Meshing-We have assumed a linear elastic and isotropic behavior of the unit cell. In addition, we have assumed an imperfect bond between the resin tags and tubule walls, such that there is insignificant stress transfer between these two components. Mechanical loads under function are characterized by low amplitude relative to material strength and linear elasticity is a reasonable assumption for performing stress distribution analysis. The elastic properties for different phases in the unit cell are taken from the literature [4,11,12]. Since the adhesive is a viscoelastic material [13], the effective elastic modulus of the adhesive is dependent on the loading rate. In our finite element calculation, we simulate the condition in which the load is applied at a relatively fast rate hence the elastic modulus of the adhesive corresponds to the value reported for a high loading rate [13]. Material properties for the different material components are given in Table 1.

Free meshing is used to generate the mesh. This type of meshing is not constrained by the geometry hence we can mesh volumes with sharp edges and corners with minimum element distortion and better accuracy. In addition, convergence studies were performed to minimize the effect of element size. Different components in the $3 \mathrm{~d}$ unit cell are meshed using 10 node parabolic tetrahedron elements. The size of the elements used for meshing depends upon the phase. Partially demineralized dentin and the proximal hybrid layer were meshed with an element size of $0.1 \mu \mathrm{m}$, the adhesive tag and peritubular dentin were meshed with $0.25 \mu \mathrm{m}$ element size, and the other components were meshed with $0.5 \mu \mathrm{m}$ element size. In the final discretized models, the total number of nodes was 575705 and total number of elements was 409345.

\section{Parametric Study}

Parametric studies were performed on the computational unit cell to investigate the effect of changes in the physical characteristics of the d-a interface, including the thickness and the material properties of the hybrid layer. To study the effect of the d-a interface microstructure variation we have developed the following four models: Model 1: Graded Hybrid Layer- in which the hybrid layer is assigned a linearly decreasing elastic modulus. The hybrid layer elastic modulus is taken to be $4 \mathrm{GPa}$ close to the adhesive layer and $1.75 \mathrm{GPa}$ at the interface with the partially demineralized dentin; Model 2: Uniform Hybrid Layer- in which the hybrid layer is assigned a uniform elastic modulus; Model 3: Thin Graded Hybrid layer- in which the hybrid layer is assigned linearly decreasing properties but its thickness is taken to be $5 \mu \mathrm{m}$, which is half that of Model 1; Model 4: Without Partially Demineralized Dentin- to investigate cases in which the etching process results in an absence of the partially demineralized dentin zone. A total of 8 parametric models were developed, 4 models for the stress controlled case and 4 models for the strain controlled case.

\section{Calculation of Stress Concentration Factors}

Stress concentration factor is defined as the ratio of highest stress experienced by the individual material components divided by the global stress which is applied on the d-a interface. In the FE calculations, the highest stress can occur in elements that are in narrow locations where several material components join together. These locations typically occupy an extremely small volume of the material component (one element) and, therefore they are not considered to be representative of the stress concentration of the material. To obtain a representative stress concentration factor, the top 5\% of high stress experiencing elements of the particular component were considered. Top 5\% data ensured that the stress concentration factor had a narrow distribution with a coefficient of variation of less than $10 \%$. Out of this 
top $5 \%$ data, a $2 \sigma$ (where $\sigma=$ standard deviation) criteria was used to discard any high stress elements which could have resulted from geometrical artifacts. The remaining elements were averaged to obtain the stress concentration in the particular material component. The stress concentration factor, denoted by $f^{\mathcal{c}}$, was then obtained as:

$$
f^{c}=\frac{\sigma_{l}^{c}}{\sigma_{g}}
$$

where $\sigma_{l}^{c}=$ average peak stress in the material component, $c$, and $\sigma_{g}=$ applied global stress on the unit cell top surface. The stress concentration factors for stress and strain controlled cases are shown in Table 2 for the four FE models considered in this work.

\section{S-N Curves for Different Material Components of d-a Interface}

The S-N (stress amplitude versus number of cycles to failure) curves for the different material components are critical for the reliability of the fatigue life prediction of the d-a interface. Generally, there is a lack of this type of data for the various material components found at the d-a interface. To demonstrate our methodology for fatigue life prediction, we have only considered the following three components: dentin, exposed collagen and adhesive. There is limited S-N data available for the components that comprise the composite/adhesive/tooth interfaces. Other components such as the hybrid layer and composite should be considered, but this requires the availability of S-N data.

The S-N curve for dentin was obtained from the literature $[14,15]$. This data was measured at frequencies of 2,10 and $20 \mathrm{~Hz}$ and a stress ratio $\left(R^{\mathrm{s}}\right)$ of 0.1 . The stress ratio $\left(\mathrm{R}^{\mathrm{s}}\right)$ is defined as a ratio of the minimum to the maximum stress during a cycle. Collagen S-N data were based upon experiments for type I collagen fibers from wallaby tail tendons [16]. These experiments were performed by keeping the minimum stress constant while increasing the maximum stress to obtain the data at different stress amplitudes. Thus the stress ratio varied from 0.57 to 0.14 for small to large stress amplitudes. If the fatigue tests were performed at the constant stress ratio of 0.1 then higher fatigue life would be expected at small stress amplitudes (see for example Fig. 11 of [17] that gives the effect of stress ratio on S-N curves for unidirectional carbon/epoxy composites). In this case, the measured curve will be primarily affected in the small stress amplitude regime, however for our proof-ofconcept calculations no modification was applied to the data. The S-N data for dentin adhesive was obtained from the experiments performed at a stress ratio of $\mathrm{R}^{\mathrm{s}}=0.1$ and frequency of $5 \mathrm{~Hz}[13]$.

The S-N curves for the three components, i.e. dentin, exposed collagen and adhesive, are fitted with a power law given by the following formula:

$$
\sigma^{c}=A\left(N_{f}\right)^{B}
$$

where A and B are fitting constants and superscript $\mathrm{c}$ refers to material components. The experimental data along with the fitted curves are shown in Figure 4, where for collagen $\mathrm{A}=52 \mathrm{MPa}, \mathrm{B}=-0.105$ and $\mathrm{R}^{2}=0.67$, for dentin $\mathrm{A}=102 \mathrm{MPa}, \mathrm{B}=-0.073$ and $\mathrm{R}^{2}=0.71$, and for adhesive $\mathrm{A}=62 \mathrm{MPa}, \mathrm{B}=-0.056$ and $\mathrm{R}^{2}=0.90$.

\section{Calculation of S-N curve of d-a Interface}

For fatigue life prediction of the dentin-adhesive interface, the stress that developed in the different material components were compared against S-N (stress-cycle) curves corresponding to each component of the d-a interface. When a global stress is applied on the computational unit cell of the $d$-a interface, different components of the d-a interface 
experience different maximum stresses as seen from Table 2. For example, the stress concentration in the adhesive tags varied from 1.16 to 1.50 times the applied load under the stress controlled conditions. The overall fatigue life of the $d$-a interface is governed by the fatigue life of the component with the shortest fatigue life at the local level. Thus by using $\mathrm{S}-\mathrm{N}$ curves for the different components and combining them with the stresses from the linear elastic FE models, we can estimate the overall fatigue life of the d-a interface.

To obtain the master S-N curve for the d-a interface, we first establish the strength and the endurance limit of the d-a interface as these properties determine the maximum and minimum stress amplitude over which the S-N curves need to be computed. Strength is defined as stress at which the material will last for only 1 cycle under constant amplitude stress loading. The endurance limit, on the other hand, is defined as the stress amplitude at which the material will not fail under constant amplitude cyclic stress loading for an arbitrarily large number of cycles. The number of cycles in our methodology is taken to be $10^{6}$. The data from Figure 4 are used to find the local strength and endurance limits for the three material components. The local strength is then divided by the stress concentration factors from Table 2 to obtain the factored strength and endurance limit for the three material components as shown in Table 3. The lowest values define the global strength and endurance limit of the d-a interface as tabulated in Table 3. It is noteworthy that the strength of the d-a interface is controlled by either the adhesive or the dentin, even though the exposed collagen is the weakest component. Once the strength and endurance limits of the $\mathrm{d}$-a interface are determined, the master S-N curve for the d-a interface may be computed as summarized below:

Step 1 A global stress is applied to the d-a interface corresponding to a stress amplitude, defined as $\left(\sigma_{\max }-\sigma_{\min }\right) / 2$, that would typically be applied in a fatigue life determination experiment. Using equation 1 and the FE computed stress concentration factors given in Table 2, the local stress-levels in the individual material components of the d-a interface are calculated.

Step 2 The number of cycles to failure for the individual material components are computed by inverting the S-N relationship given in Eq 2 as:

$$
N_{f}^{c}=10^{\frac{\log (A)-\log (\sigma a)}{B}}
$$

and using the local stress-levels from step 1 . The material component that requires the least number of cycles to failure will be the component that governs the d-a interface failure at the specific global stress.

Steps 1 and 2 are applied repeatedly for stress amplitudes between the strength and the endurance limit to construct the overall $\mathrm{S}-\mathrm{N}$ curve. We illustrate the procedure through two example calculations for Model 1 under stress controlled loading. In the first example, a global stress of $35 \mathrm{MPa}$ is applied. Thus, using the stress concentration factors from Table 2, the local stress levels and the number of cycles to failure at the different material components are calculated as:

$\sigma_{l}^{\text {adhesive }}=52.5 \mathrm{MPa}, N_{f}^{\text {adhesive }}=18 ; \sigma_{l}^{\text {exposed }- \text { collagen }}=36.4 \mathrm{MPa}, N_{f}^{\text {exposed }- \text { collagen }}=28$; and $\sigma_{l}^{\text {dentin }}=45.5 \mathrm{MPa}, N_{f}^{\text {dentin }}=66951$. In this case, the overall fatigue life is governed by the adhesive, which has the lowest number of cycles to failure. In the second example, a global stress of $30 \mathrm{MPa}$ is applied and the corresponding number of cycles to failure is found to be: $N_{f}^{\text {adhesive }}=271 ; N_{f}^{\text {exposed-collagen }}=120 ;$ and $N_{f}^{\text {dentin }}=5.61 \times 10^{5}$. In this second case, the exposed collagen will fail first because it has the lowest number of cycles to failure. These examples 
clearly show that under different applied stress amplitudes, different components will govern the fatigue life behavior of the d-a interface.

\section{RESULTS AND DISCUSSION}

\section{Stress Analysis of d-a Interface}

For all the finite element models of the $\mathrm{d}$-a interface the maximum principle stresses were used as the critical stress. Figure 5(A) shows the maximum principle stresses in the four different models under the stress controlled loading. High stresses, approximately 1.5 times that of the applied stress, can be seen in the adhesive tags because of softer material properties and geometry of the adhesive tags. However, stress distribution and stress values differ from model to model. Stresses in the restorative dental composite, adhesive layers and intertubular dentin were fairly uniform and almost equal to the applied stress. Peritubular dentin also experiences high stress but not as high the stress noted in the adhesive tags. Most of the stresses seen in unit cells were tensile with very little compressive stress.

If we compare the four different models in Figure 5(A), the unit cell with uniform hybrid layer has less stress concentration in the adhesive tags as compared to the unit cell with a graded hybrid layer. These differences reflect the homogeneous elastic properties throughout the depth of the uniform hybrid layer. When the hybrid layer is thin (Figure 5(A) model 3), there is a reduction in stress values as compared with a thick graded hybrid layer. The stress distributions in the unit cell that does not contain a zone of partially demineralized dentin (Figure 5(A) model 4) and the unit cell with a graded hybrid layer are almost identical. Further, the stress distribution in the exposed collagen of the thick graded hybrid layer (Figure 5(D) model 1) and the thin graded hybrid layer (Figure 5(D) model 3) is similar. The exposed collagen in the unit cell with no partially demineralized dentin (Figure 5(D) model 4) has slightly lower stresses as compared to the unit cell with a graded hybrid layer. Lastly stress distribution in the intertubular dentin and peritubular dentin (Figure $5(\mathrm{C})$ ) is almost the same in all four models.

Stress controlled loading (Figure 5(A)) showed higher stress concentration in adhesive tags as compared to dentin and other phases, hence in this case adhesive tags are more prone to failure due to relatively higher stress. On the other hand under strain controlled (Figure 5(B)) loading, because of the nature of the boundary conditions, the stresses in the adhesive and adhesive tags are less as compared to stresses in the dentin and composite. Hence, in this case, the adhesive and adhesive tags are experiencing stress shielding. Other than the above mentioned difference the results of the stress controlled loading were the same as the strain controlled loading.

Results from the stress and strain finite element model of the d-a interface suggest that the mean stress experienced by the adhesive tag under masticatory function could vary from 1.5 to 0.35 times the applied load. Moreover a d-a interface that possesses a thin hybrid layer with uniform material properties experiences smaller and more uniform stresses as compared to a thick hybrid layer with graded properties.

In this study only axial loading is considered for the unit cell. Similar calculations may be performed under shear loads. This will be done in our future studies. . Residual shrinkage stresses present in composite restorations are also expected to impact fatigue. These stresses must be accounted for in models that provide fatigue prediction of the restored tooth. In the methodology presented in this paper such stresses could be incorporated provided that the stresses can be computed at the local scales. The shrinkage process and its consequences are complex; understanding the impact of shrinkage on fatigue requires detailed investigations using the global-local methodology provided in this paper. Such a study is out of the scope 
of the present paper, especially since only imperfect empirical information is available currently.

\section{Fatigue Life Prediction of d-a Interface}

For the fatigue life prediction of the d-a interface it is very important to have empirically determined S-N curves for the different material components. Due to the lack of data only three phases, exposed-collagen, dentin and adhesive were used in this study.. Stress concentration for the three different phases was calculated based upon the finite element analysis. Moreover, the methodology for fatigue life prediction in this study is based upon isolated computational unit cell having periodic boundary conditions. Under clinical function, the unit cell for the d-a interface will have both strain and stress boundary conditions. Hence it is important to consider two extreme cases of loading which can give upper and lower bounds in terms of fatigue life for the d-a interface

We have demonstrated the methodology for the two extreme cases of geometries: 1) a wellinfiltrated hybrid layer of uniform mechanical properties, and 2) a poorly-infiltrated hybrid layer of graded mechanical properties. The d-a interface thickness is $15 \mu \mathrm{m}$ for both cases. Under the stress controlled loading, the adhesive has the shortest fatigue life at higher stress amplitudes, whereas, at lower stress amplitudes, either exposed-collagen or the adhesive could fail depending upon the microstructure of the hybrid layer. On the other hand, under strain controlled loading dentin has the shortest fatigue life at higher stress amplitudes. Under strain loading the adhesive does not fail because of stress shielding.

All the S-N data for the d-a interface under stress and strain loading along with a measured $\mathrm{S}-\mathrm{N}$ curve at $\mathrm{R}^{\mathrm{s}}=0.1[18]$ for the dentin-composite interface are shown in Figure 6. If we compare the four S-N curves, the d-a interface with graded hybrid layer under stress controlled loading has the lowest fatigue life which gives the lower limit for the fatigue behavior of the d-a interface. On the other hand, the d-a interface with uniform hybrid layer under strain controlled loading has the longest fatigue life which gives the upper bound for the fatigue behavior of the d-a interface. The measured curve falls in the stress controlled region because Staninec et al [18] has performed the experiment on small rectangular beam samples which have smaller number of unit cells as compared to the actual d-a interface. Fewer unit cells mean less constraint in the lateral direction. Hence the d-a unit cell in their study could be experiencing stress boundary conditions without constraint in the lateral direction, therefore the measured S-N curve falls in the region of stress controlled loading. It is noteworthy that the predicted failure location is in the adhesive tags in the proximity of the hybrid layer, and at no stress-level does the failure proceed through the dentin or the composite. This failure pathway agrees with the visual microscopic examination of the failed samples by Staninec et al [18].

Under function, dentin adhesives are subjected to both chemical and mechanical stresses and the interplay between these stresses can result in an alteration of the adhesive mechanical properties with time. The mechanical property change results from a variety of mechanisms including (i) proliferation of surface and subsurface flaws and (ii) change in the chemical nature of the polymer in the form of either strain hardening, embrittlement, crystallization or plasticization. The change in adhesive properties can have a significant effect upon the mechanical performance and durability of the d-a interface. We further note that although we have only considered 3 components to illustrate our method, other components, such as the hybrid layer can be readily incorporated once $\mathrm{S}-\mathrm{N}$ data becomes available. In our future work, we will incorporate the effect of combined chemo-mechanical stress as well as the fatigue data of other components that comprise this complex material/tissue interface. . 


\section{Conclusion}

We have developed a methodology for the prediction of fatigue life of the dentin-adhesive (d-a) interface. Based upon stress analysis using the $3 \mathrm{~d}$ FE model, we have shown that different material components of the d-a interface suffer different maximum stresses. As a result, the overall fatigue life of the d-a interface is governed by the fatigue life of the material component with the shortest fatigue life under a given loading condition. Therefore by using the S-N curves for different material components and combining them with the micromechanical stress analysis, the overall fatigue life of the d-a interface can be estimated.

Stress analysis using the $3 \mathrm{~d}$ FE model of the d-a interface indicated that under both stress and strain controlled loading conditions, stress distribution is largely effected by the material properties of the hybrid layer and the physical characteristics of the dentin-adhesive interface. From the FE calculations it can be concluded that the d-a interface with a thin hybrid layer that possesses uniform material properties, will experience the least amount of stress concentration under both loading conditions. The fatigue life of the d-a interface was found to be a function of the type of loading, the properties of the adhesive and the microstructure of the d-a interface. The S-N curve of the d-a interface does not follow one function, rather different regions of the S-N curve are governed by the different material components of the d-a interface. In both the stress and strain controlled cases the d-a interface with uniform hybrid layer showed longer fatigue life as compared to the $d$-a interface with a graded hybrid layer.

\section{Acknowledgments}

The authors gratefully acknowledge research support from NIH/NIDCR grants DE14392 (PS) and 3R01DE014392-08S109 (PS).

\section{References}

1. Misra A, Spencer P, Marangos O, Wang Y, Katz JL. Parametric Study of the Effect of Phase Anisotropy on the Micromechanical Behavior of dentin-adhesive Interface. Journal of Royal Society Interface. 2005; 2:145-57.

2. Wang Y, Spencer P. Quantifying adhesive penetration in adhesive/dentin interface using confocal Raman microspectroscopy. J Biomed Mater Res. 2002; 59(1):46-55. [PubMed: 11745536]

3. Wang Y, Spencer P. Overestimating hybrid layer quality in polished adhesive/dentin interfaces. J Biomed Mater Res A. 2004; 68(4):735-46. [PubMed: 14986328]

4. Katz JL, Bumrerraj S, Dreyfuss J, Wang Y, Spencer P. Micromechanics of the dentin/adhesive interface. J Biomed Mater Res. 2001; 58(4):366-71. [PubMed: 11410894]

5. Misra A, Spencer P, Marangos O, Wang Y, Katz JL. Micromechanical Analysis of Dentin/Adhesive Interface by the Finite Element Method. J Biomed Mater Res Part B: Appl Biomater. 2004; 70B: 56-65. [PubMed: 15199584]

6. Singh, V. Viscoelastic and fatigue properties of dental adhesives and their impact on dentinadhesive interface durability. Lawrence: University of Kansas; 2009.

7. Wieliczka DM, Kruger MB, Spencer P. Raman imaging of dental adhesive diffusion. Appl Spectrosc. 1997; 51:1593-6.

8. Wieliczka DM, Spencer P, Kruger MB. Raman mapping of the dentin/adhesive interface. Appl Spectrosc. 1996; 50:1500-4.

9. Dejak B, Mlotkowski A, Romanowicz M. Finite element analysis of stresses in molars during clenching and mastication. J Prosthet Dent. 2003; 90(6):591-7. [PubMed: 14668761]

10. Nasser, SN.; Hori, M. Micromechanics: Overall Properties of Heterogeneous Materials. Elsevier; 1999. 
11. Katz JL, Spencer P, Nomura T, Wagh A, Wang Y. Micromechanical properties of demineralized dentin collagen with and without adhesive infiltration. J Biomed Mater Res A. 2003; 66(1):120-8. [PubMed: 12833438]

12. Kinney JH, Balooch M, Marshall GW, Marshall SJ. A micromechanics model of the elastic properties of human dentine. Arch Oral Biol. 1999; 44(10):813-22. [PubMed: 10530914]

13. Singh V, Misra A, Marangos O, Spencer P, Park J, Ye Q, et al. Viscoelastic and Fatigue Properties of Model Methacrylate Based Dentin Adhesives. Journal of Biomedical Material Research Part B. 2010

14. Nalla RK, Imbeni V, Kinney JH, Staninec M, Marshall SJ, Ritchie RO. In Vitro Fatigue Behavior of Human Dentin with Implications for Life Prediction. Journal of Biomedical Material Research. 2003; 66A:10-20.

15. Nalla RK, Kinney JH, Marshall SJ, Ritchie RO. On the in vitro Fatigue Behavior of Human Dentin: Effect of Mean Stress. Journal of Dental Research. 2004; 83(3)

16. Wang XT, Ker RF, Alexander RM. Fatigue Rupture of Wallaby Tail Tendons. The Journal of Experimental Biology. 1995; 198:847-52. [PubMed: 9244805]

17. Chen HS, Hwang SF. Accelerated Fatigue Properties of Unidirectional Carbon/Epoxy Compsite Materials. Journal of Polymer Compsities. 2006; 27:138-46.

18. Staninec M, Kim P, Marshall GW, Ritchie RO, Marshall SJ. Fatigue of Dentin-Composite Interface with Four-Point Bend. Dental Materials. 2008; 24:799-803. [PubMed: 17996931] 


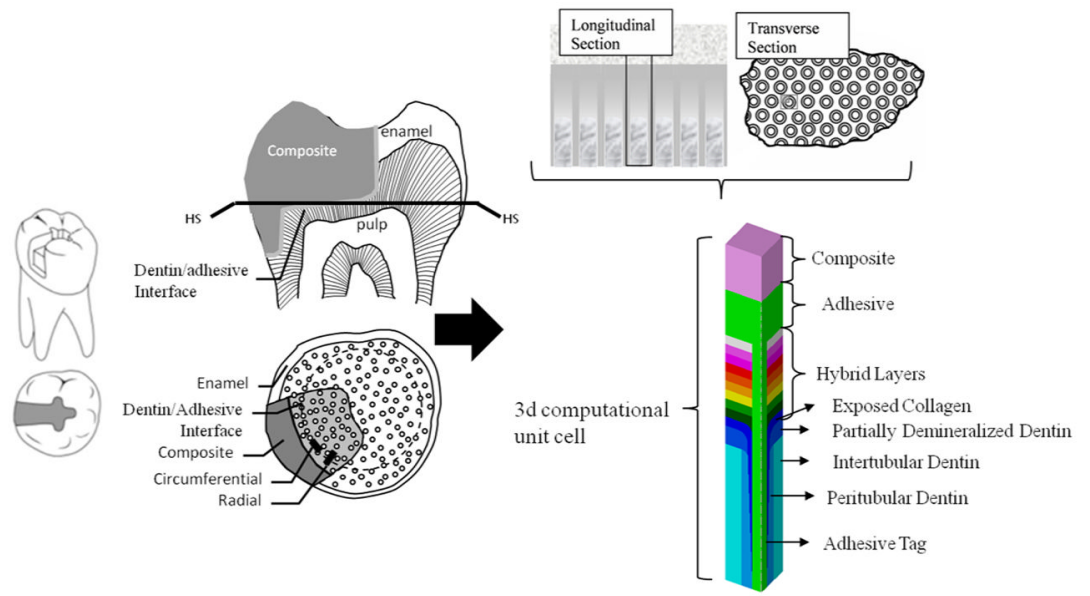

Figure 1.

Schematic of the d-a interface and the idealized computational unit cell. 


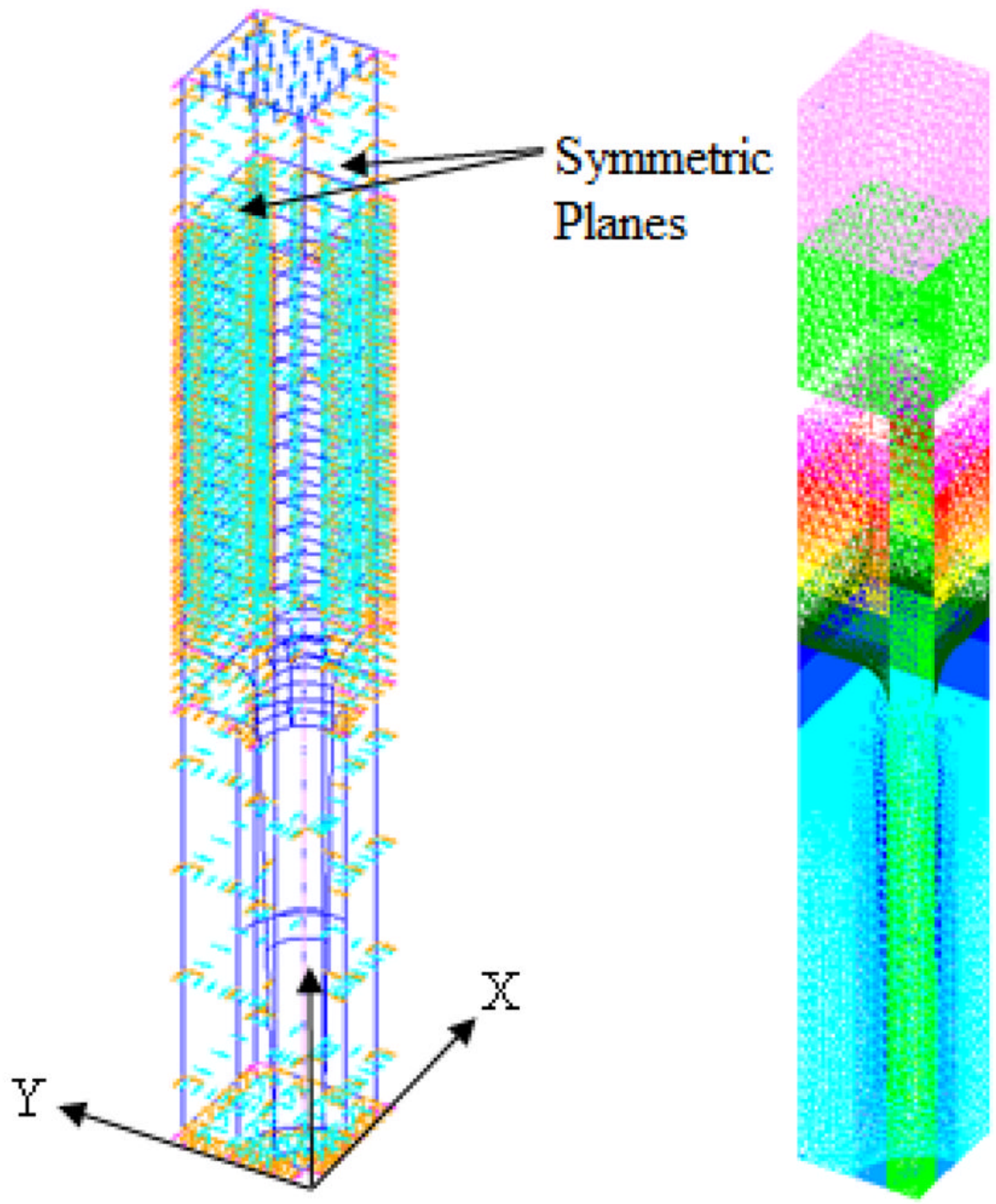

Figure 2.

A3d finite element model showing boundary conditions and mesh of the computational unit cell. 

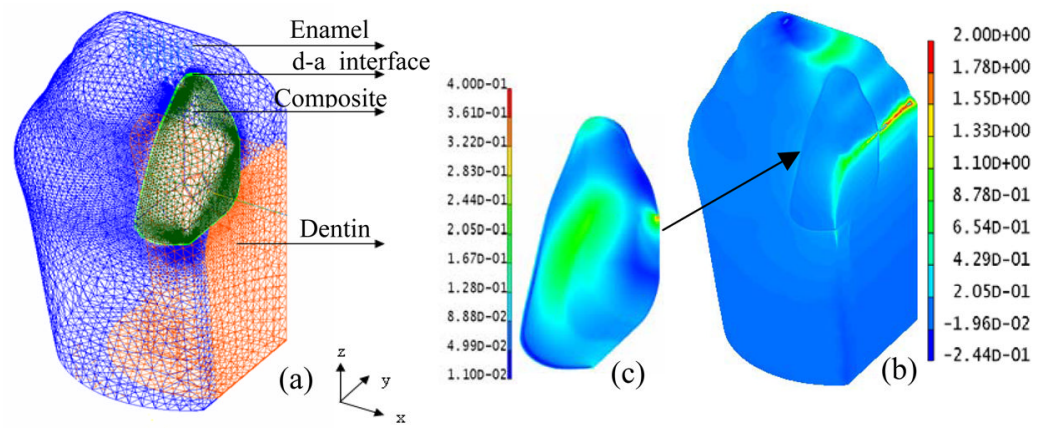

Figure 3.

Global stress analysis of a restored tooth: (a) 3d finite element model, (b), stress distribution in the restored tooth and (c) stress distribution in the d-a interface. 


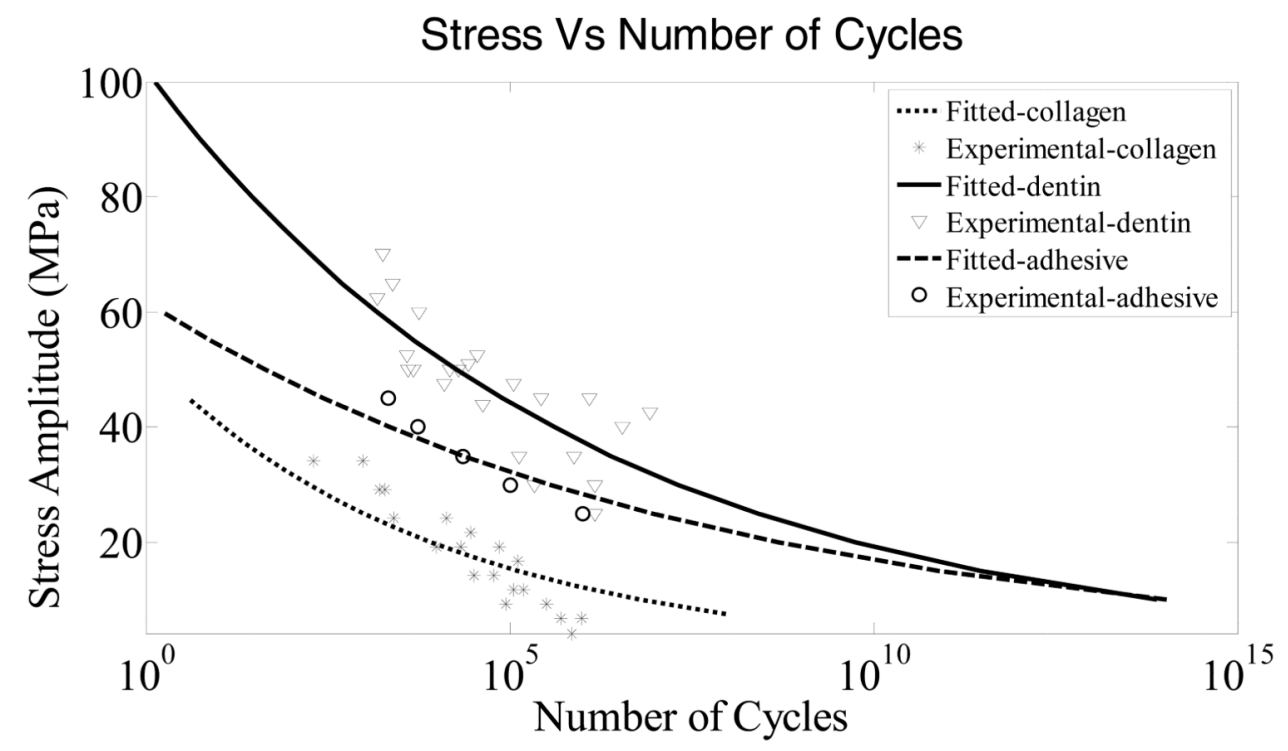

Figure 4.

$\mathrm{S}-\mathrm{N}$ curves for different material components of d-a interface. 

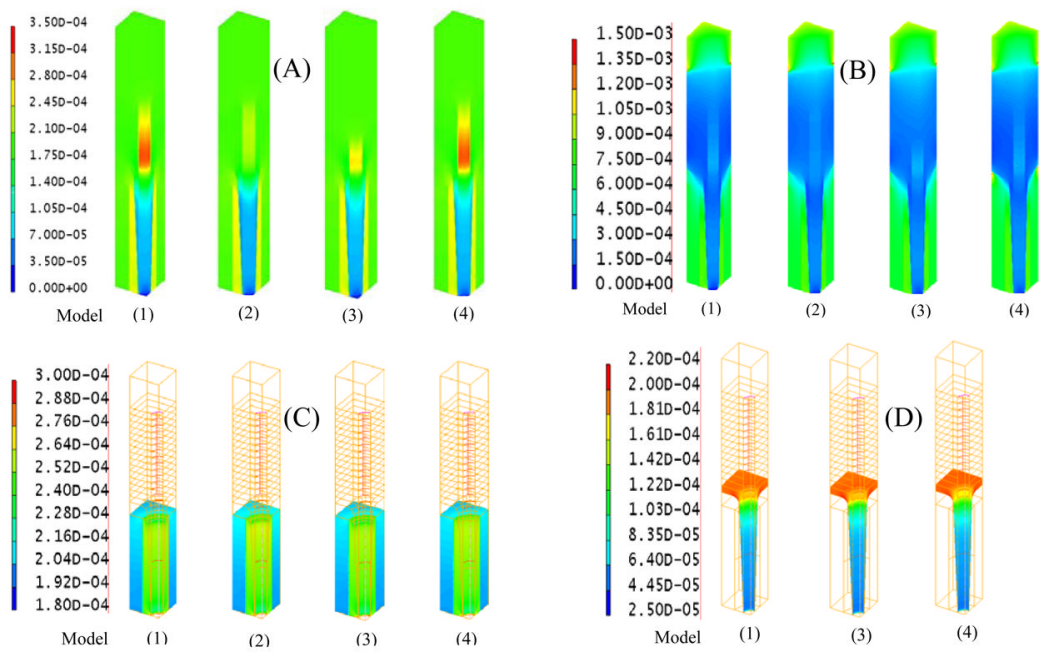

Figure 5.

Maximum principle stress distributions $\left(\times 10^{5} \mathrm{MPa}\right)$ in the computational unit cell (A) under stress controlled loading and (B) under strain controlled loading. The stress distributions in peritubular and intertubular dentin region (C), and exposed collagen region (D), respectively are for case A. 


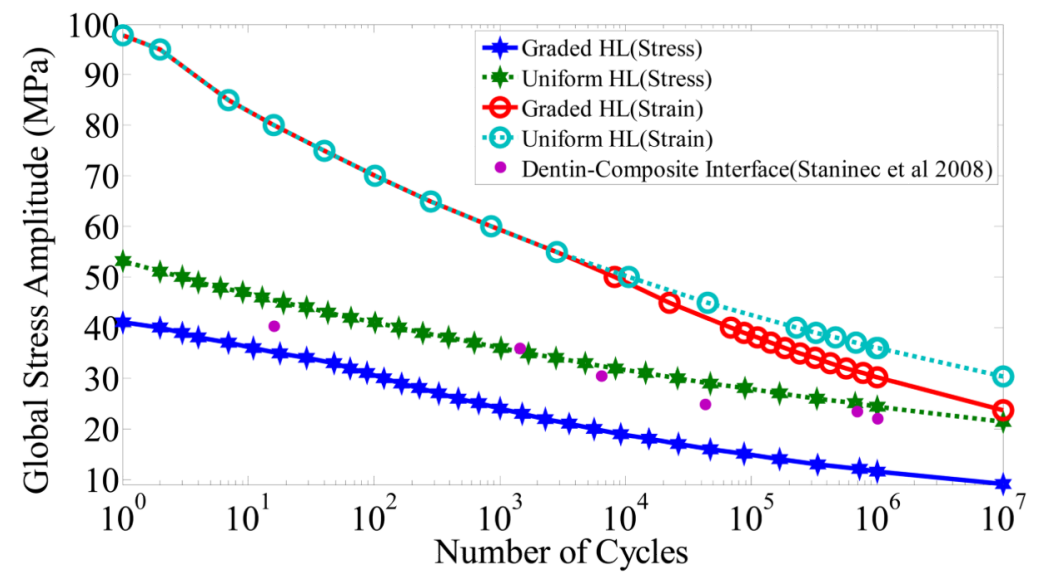

Figure 6.

Master S-N curves for $\mathrm{d}$-a interface under different loading conditions along with the measured data. 
Table 1

Material property of different components of $d$-a interface

\begin{tabular}{|l|l|l|}
\hline Material & Young's Modulus, E(GPa) & Poisson's Ratio, $\boldsymbol{v}$ \\
\hline Composite & 30 & 0.29 \\
\hline Adhesive & 5 & 0.29 \\
\hline Peritubular Dentin & 26 & 0.29 \\
\hline Intertubular Dentin & 20 & 0.29 \\
\hline Partially Demineralized Dentin & 13 & 0.29 \\
\hline Hybrid Layer-1-10 & $4-1.75$ & 0.29 \\
\hline
\end{tabular}




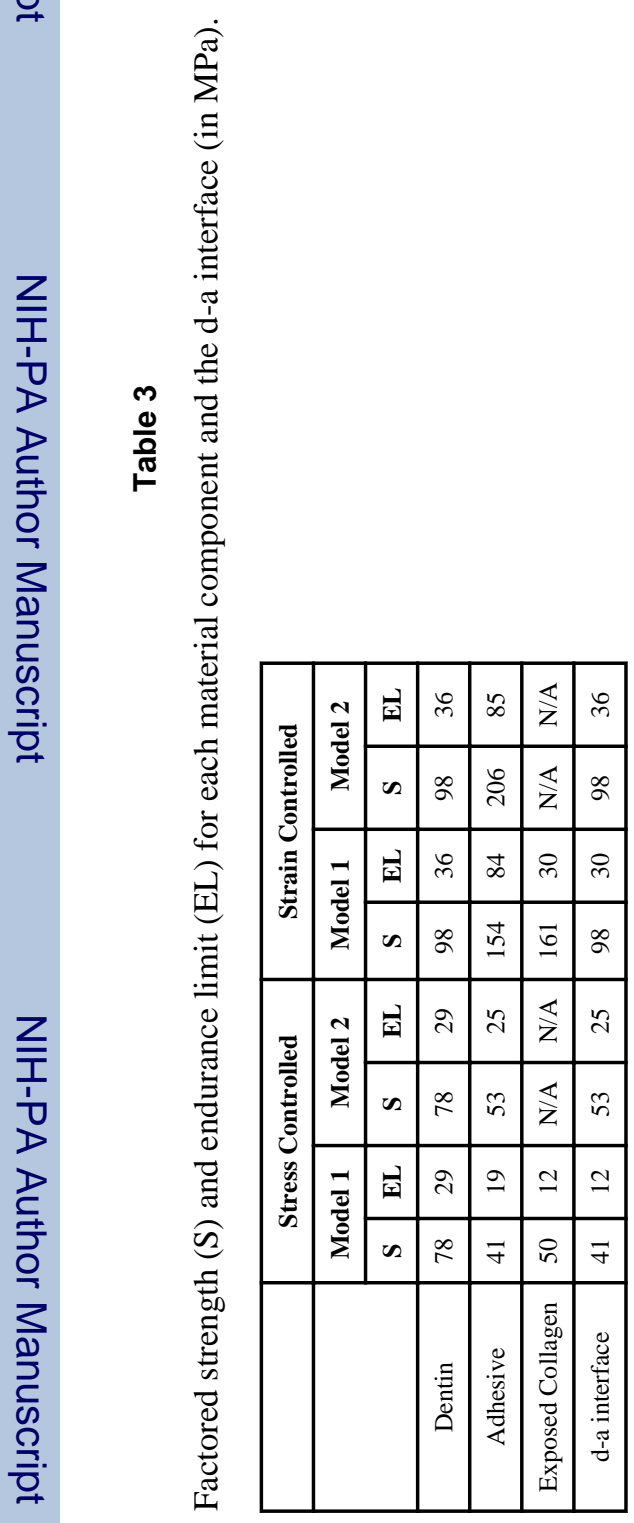

Dent Mater. Author manuscript; available in PMC 2013 May 20. 